\title{
Aerobic responses of prepubertal boys to two modes of training
}

\author{
Craig A Williams, Neil Armstrong, Julian Powell
}

\begin{abstract}
Objective-To investigate the effects of two contrasting eight week training programmes on the aerobic performance of 39 prepubescent boys (mean age 10.1 years).

Methods-All boys were volunteer subjects from three city schools and the schools were matched by a health related behaviour questionnaire. All of the boys were assessed as Tanner stage one for genitalia and pubic hair development. Criterion laboratory tests included peak $\dot{\mathbf{V}} \mathrm{O}_{\text {, }}$ as assessed by an incremental discontinuous treadmill test to voluntary exhaustion. Submaximal measurements of heart rate, minute ventilation $\left(\dot{V}_{E}\right)$ and $\dot{V}_{O_{2}}$ were also recorded during the treadmill test. One of the schools provided the control group $(n=14)$, and boys from the other schools followed two contrasting training programmes. The first was a sprint interval running programme $(n=$ 12) comprising 10 second and 30 second sprints, and the second a continuous cycle ergometer programme $(n=13)$ maintaining a heart rate in the range $80-85 \%$ of maximum for 20 minutes on a Monark cycle ergometer. After eight weeks training three times a week, the three groups were retested.
\end{abstract}

Results-There were no significant differences in peak $\dot{\mathrm{V}}_{2}(\mathrm{p}>0.05)$ with training in either of the groups. Neither were there significant changes in any of the submaximal variables $\dot{V}_{2}, \dot{V} E$, or heart rate $(\mathrm{p}>0.05)$.

Conclusion-The findings of this study indicate that neither eight week sprint interval running nor continuous cycle ergometer training programmes significantly improve maximal or submaximal indicators of the aerobic performance of prepubertal boys.

(Br F Sports Med 2000;34:168-173)

Keywords: oxygen uptake; aerobic performance; training; prepubescent; boys

Exercise training studies with prepubertal boys have produced equivocal results. Some studies have reported training induced improvements in peak $\dot{\mathrm{V}}_{2},{ }^{1}{ }^{2}$ whereas others have observed no significant changes in peak $\dot{\mathrm{V}}_{2}$ after training. ${ }^{3} 4$ Reasons for the lack of information on responses of prepubertal boys to training include poor experimental design, ${ }^{5}{ }^{6}$ high attrition rate, and pooling of data on boys and girls. ${ }^{4} 7$

It has been hypothesised that a maturational threshold $^{8}$ or a trigger point ${ }^{9}$ may exist below which the effects of training will be minimal. Indeed Gilliam and Freedson ${ }^{4}$ stated, "it appears that a maturational factor may determine a child's potential for physiologic alterations to occur consequent to physical training" (p 77). Others have argued that there is no evidence that the training response of prepubescent boys is less than in older boys. ${ }^{10}$ Two recent systematic reviews of the literature have concluded that, although young boys will respond to appropriate training programmes with increases in peak $\dot{\mathrm{V}}_{2}$, the size of the changes may be less than those expected in older youths and adults. ${ }^{5}$ However, few studies have assessed maturational status and reported the responses of prepubescent boys to carefully controlled and well defined exercise training. ${ }^{1} 7$

Most training studies have used continuous exercise as a method of improving aerobic performance. However, studies that have investigated physical activity patterns of young people have shown that children seldom experience five, 10 , or 20 minute periods of sustained exercise. ${ }^{11} \mathrm{~A}$ recent study by Bailey $e t$ al ${ }^{12}$ used direct observation of 6-10 year old children over nine four hour observation periods. The authors concluded that the children were engaged in short vigorous bouts of physical activity, usually lasting no longer than 15 seconds, interspersed with varying levels of low to moderate activity. Hence it would appear that the transitory nature of children's physical activity patterns may be well suited to a high intensity intermittent training programme. To our knowledge, no published study has examined the effects of a well controlled intermittent exercise programme on the subsequent aerobic performance of prepubertal boys.

Data on the aerobic training responses of prepubertal boys are sparse, and most studies have not assessed maturation or monitored carefully the training modality, especially the intensity of exercise. It is important to ascertain
Accepted for publication 8 December 1999 
and quantify these issues, as both may have a major impact on the training response. Identification of the magnitude of the aerobic response to intermittent and continuous exercise of children engaged in these types of sporting activities is essential. The aim of the study was therefore to investigate the effect of two modes of training on the aerobic performance of prepubertal boys.

\section{Methods}

Forty five boys (mean (SD) age 10.1 (0.2) years) from three city schools volunteered to participate in the project. The three schools were chosen because they are similar in size, follow the United Kingdom National Curriculum in physical education, and had been matched using a health related behaviour questionnaire in a recent longitudinal study involving all city schools. ${ }^{13}$ Before the beginning of the study, ethical approval of procedures and the informed consent of both the children and their parents were obtained.

For habituation purposes, each school group visited the laboratory separately to become fully acquainted with the test procedures. All volunteers were classified as Tanner stage one according to genitalia and pubic hair development ${ }^{14}$ by a nurse resident in the laboratory and experienced in the use of the technique. The schools were then randomly assigned to one of three groups, the sprint interval running training (SIR) group, the continuous cycle ergometer training (CCE) group, or the control (C) group.

After this visit, the children revisited the laboratory on two further occasions, one before training and one after training. Before the peak $\dot{\mathrm{V}}_{2}$ test, body mass to the nearest $0.1 \mathrm{~kg}$, using an Avery balance beam (Avery, Birmingham, $\mathrm{UK}$ ), and stature to the nearest $0.01 \mathrm{~m}$, using a Holtain stadiometer (Crymych, Dyfed, Wales, UK), were measured.

The peak $\dot{\mathrm{V}}_{2}$ test consisted of an incremental discontinuous treadmill test to voluntary exhaustion. The test was conducted on a motorised treadmill (Woodway, Cranlea Ltd, Birmingham, UK). The test consisted of a warm up at a belt speed of between 1.82 and $1.96 \mathrm{~m} / \mathrm{s}(6.5$ and $7 \mathrm{~km} / \mathrm{h})$ for three minutes followed by a rest period. The first running period consisted of three minutes at a speed of $1.96 \mathrm{~m} / \mathrm{s}(7 \mathrm{~km} / \mathrm{h})$ at a $0 \%$ gradient. Then the speed was increased to $2.24 \mathrm{~m} / \mathrm{s}(8 \mathrm{~km} / \mathrm{h})$ for a further three minutes; thereafter this speed was held constant but the treadmill gradient was raised by $2.5 \%$ before each subsequent three minute period. Peak $\dot{\mathrm{V}}_{2}$ was accepted as a maximal index if there were visual signs of exhaustion despite coaxing to continue, if the heart rate was levelling off at about 200 beats/ min, and the respiratory exchange ratio (RER) was greater than unity. Maximal minute ventilation $\left(\dot{V}_{E}\right)$ was also noted at the end of the test. Throughout the test, inspired and expired gases were monitored continuously using an Oxycon Sigma metabolic cart (Cranlea Ltd). The system was calibrated before each test with gases of known oxygen and carbon dioxide concentration. Heart rate was determined continuously using an electrocardiograph (Rigel, Morden, Surrey, UK).

Lactate at peak $\dot{\mathrm{V}}_{2}$ was recorded three minutes after the cessation of the $\dot{\mathrm{V}}_{2}$ test using a fingertip capillary blood sample (about $25 \mu \mathrm{l}$ ). Each blood sample was immediately analysed using a whole blood analyser (YSI 2300 Stat Plus; Yellow Springs Instruments, Yellow Springs, Ohio, USA). The analyser self calibrated with a known concentration of lactate every five samples, and the calibration was checked regularly against commercially prepared standards of verified concentration. Submaximal indices of aerobic performance including $\dot{\mathrm{V}}_{2}, \dot{\mathrm{V}} \mathrm{E}$, and heart rate were collected during the first three running stages of the peak $\dot{\mathrm{V}} \mathrm{O}_{2}$ test.

\section{TRAINING PROGRAMMES}

Sprint interval running

Before the beginning of the training, each volunteer was asked to sprint as fast as possible and to cover as much distance as they could in a 10 second and a 30 second run. This process was repeated several times, and it was emphasised that each boy should run as hard and as fast as possible and not to try to pace themselves. The greatest distance that the boys covered was recorded and from the result each boy was set a target which they had to try to beat during the training sessions. The target was continuously changed for each boy based on the highest recorded distance from the previous day's training session or their greatest recorded distance recorded in the programme, whichever was the higher score. All training took place on Mondays, Wednesdays, and Fridays at the same time on the school playground. A series of $25 \mathrm{~m}$ lanes interspersed with cones at $5 \mathrm{~m}$ intervals was set up. All boys ran three 10 second sprints with a 30 second rest interval between runs followed by three 30 second sprints with 90 second rest intervals. The boys worked in pairs; while one was resting the other partner recorded the distance run and entered it into a log book, then the roles were reversed. To develop progressive overload, the number of runs was increased to four after two weeks and then five and six at weeks four and six respectively. Intensity of effort was estimated from heart rates recorded by Sports Tester 3000 heart rate monitors (Polar Electro, Kempele, Finland). Because of inclement weather, three of the 24 sessions were performed indoors in the school hall. All the training sessions were monitored by the same researchers. The log book in conjunction with the heart rate data was constantly monitored to ensure consistency of effort. Twelve boys completed at least 23 of the 24 training sessions.

\section{Continuous cycle ergometry training}

The CCE group also trained on Mondays, Wednesdays, and Fridays at the same time and their training consisted of continuous cycling for 20 minutes three times a week. All the sessions were conducted on Monark cycle ergometers, and the boys maintained a heart rate between 160 and 170 beats/min (about 80- 
Table 1 Peak $\dot{V}_{\mathrm{O}_{2}}$, heart rate, $\dot{V} E$, blood lactate, and respiratory exchange ratio (RER) before (Pre) and after (Post) training.

\begin{tabular}{|c|c|c|c|}
\hline & $\operatorname{SIR}(n=12)$ & $C C E(n=13)$ & $C(n=14)$ \\
\hline \multicolumn{4}{|l|}{ Peak $\dot{\mathrm{VO}_{2}}$ (litres/min) } \\
\hline Pre & $1.84(0.2)$ & $1.80(0.3)$ & $1.92(0.2)$ \\
\hline Post & $1.91(0.3)$ & $1.93(0.3)$ & $1.97(0.2)$ \\
\hline Range of $\%$ changes & $-6.1-16.4$ & $-9.8-25.3$ & $-4.4-14.5$ \\
\hline \multicolumn{4}{|l|}{ Peak $\mathrm{V}_{2}(\mathrm{ml} / \mathrm{kg} / \mathrm{min})$} \\
\hline Pre & $54.8(5.1)$ & $54.7(9.7)$ & $56.4(8.2)$ \\
\hline Post & $53.9(7.8)$ & $57.5(7.0)$ & $56.7(8.1)$ \\
\hline Range of \% changes & $-6.9-17.0$ & $-4.4-21.8$ & $-3.4-17.5$ \\
\hline \multicolumn{4}{|l|}{ Heart rate (beats/min) } \\
\hline Pre & $205(6)$ & $204(7)$ & $200(7)$ \\
\hline Post & $203(7)$ & $201(7)$ & $196(8)$ \\
\hline \multicolumn{4}{|l|}{$\dot{\mathrm{V}} \mathrm{E}$ (litres/min) } \\
\hline Pre & $63.0(12.0)$ & $57.2(7.1)$ & $63.7(8.5)$ \\
\hline Post & $66.1(7.7)$ & $63.8(7.7)$ & $64.9(9.3)$ \\
\hline \multicolumn{4}{|c|}{ Blood lactate at peak $\dot{\mathrm{V}}_{2}(\mathrm{mmol} / \mathrm{l})$} \\
\hline Pre & $5.9(2.5)$ & $4.8(0.9)$ & $4.7(1.3)$ \\
\hline Post & $6.5(2.2)$ & $5.6(2.2)$ & $5.2(1.3)$ \\
\hline \multicolumn{4}{|l|}{ RER } \\
\hline Pre & $1.09(0.05)$ & $1.07(0.03)$ & $1.04(0.05)$ \\
\hline Post & $1.08(0.05)$ & $1.03(0.04)$ & $1.04(0.04)$ \\
\hline
\end{tabular}

Values are mean (SD). SIR, sprint interval running; CCE, continuous cycle ergometry; C, control. No significant difference between groups $(p>0.05)$.

$85 \%$ maximum heart rate). The relative intensity of training is higher than that recommended for adults, as review of the paediatric training literature suggests intensities of this magnitude are necessary for significant improvements in aerobic fitness. The heart rates were monitored using either a Sports Tester 3000 heart rate telemeter or an electrocardiograph. All sessions were rigorously supervised by a researcher. Thirteen boys completed at least 23 of the 24 training sessions.

Control

After the pretraining tests, the group was instructed to take part in normal everyday activities and informed that they were to be retested after eight weeks. A total of 14 boys successfully completed all the before and after training measurements.

STATISTICAL ANALYSIS

All data were stored and analysed using the statistical package SPSS for Windows 6.1 (SPSS Inc, Chicago, Illinois, USA). The submaximal and maximal data were analysed using a repeated measures analysis of variance. The alpha level of 0.05 was used for all statistical analyses.

\section{Results}

The mean (SD) ages of the boys in the SIR, CCE, and C groups were 10.1 (0.3), 10.1 $(0.2)$, and $10.1(0.3)$ years respectively. Their mean statures before and after training were $1.42(0.1)$ and $1.43(0.1), 1.39(0.1)$ and 1.40 (0.1), and $1.41(0.1)$ and $1.43(0.1) \mathrm{m}$ respectively, and their mean body masses before and after training were 35.5 (5.4) and 35.4 (4.7), 34.7 (10.0) and 34.7 (10.0), and 35.5 (7.1) and $35.7(7.5) \mathrm{kg}$ respectively. A repeated measures analysis of variance showed no significant differences between groups for age, stature, or body mass ( $p>0.05)$.

All children in the training groups completed at least 23 out of 24 training sessions. Table 1 shows the maximal aerobic performance data before and after eight weeks of training. It should be noted that, before the start of the study, there was no significant difference in peak $\dot{\mathrm{V}}_{2}$ between the three groups (table 1). No significant differences were found for either of the training modes $(\mathrm{p}>0.05)$ on peak $\dot{\mathrm{V}}_{2}$ when expressed in absolute terms (litres/min) or relative to body mass $(\mathrm{ml} / \mathrm{kg} / \mathrm{min})$. Similarly, no significant differences were found for $\dot{V} E$, heart rate, $\mathrm{RER}$, or lactate at peak $\dot{\mathrm{V}}_{2}$. Table 1 also shows the range of percentage increases in absolute and relative peak $\dot{\mathrm{V}}_{2}$. The highest percentage increase in peak $\mathrm{VO}_{2}$ was found in one subject from the CCE group who improved $25 \%$ in absolute terms and $22 \%$ relative to body mass.

Table 2 gives the submaximal aerobic responses before and after training. $\dot{\mathrm{V}}_{2}$ did not change significantly over the first three submaximal exercise intensities in any group during training $(p>0.05)$. Although there was a tendency for the $\dot{\mathrm{V}}_{2}$ scores to be lower after training, there was wide variability in the aerobic responses as reflected by the large standard deviation scores. Table 3 shows the scores for

Table 2 Submaximal $\dot{V}_{\mathrm{O}_{2}}(\mathrm{ml} / \mathrm{kg} / \mathrm{min})$ at the first three stages of the treadmill test before (Pre) and after (Post) training.

\begin{tabular}{llll}
\hline & $7 \mathrm{~km} / \mathrm{h}$ & $8 \mathrm{~km} / \mathrm{h}$ & $\begin{array}{l}8 \mathrm{~km} / \mathrm{h} \text { and } \\
2.5 \% \text { gradient }\end{array}$ \\
\hline SIR & $32.1(5.5)$ & $36.8(3.6)$ & $40.3(3.7)$ \\
$\quad$ Pre & $30.7(4.5)$ & $33.8(5.9)$ & $39.8(1.9)$ \\
$\quad$ Post & & & \\
CCE & $30.9(3.4)$ & $34.4(3.2)$ & $37.8(4.4)$ \\
$\quad$ Pre & $32.5(3.2)$ & $33.9(5.9)$ & $39.1(2.8)$ \\
$\quad$ Post & $34.2(3.7)$ & $36.6(4.2)$ & $40.8(2.9)$ \\
Pre & $31.7(5.3)$ & $36.4(4.5)$ & $39.2(3.0)$ \\
$\quad$ Post & & & \\
\hline
\end{tabular}

Values are mean (SD). SIR, sprint interval running; CCE, continuous cycle ergometry; C, control. No significant difference between groups $(\mathrm{p}>0.05)$.

Table 3 Submaximal heart rate (beats/min) at the first three stages of the treadmill test before (Pre) and after (Post) training.

\begin{tabular}{llll}
\hline & $7 \mathrm{~km} / \mathrm{h}$ & $8 \mathrm{~km} / \mathrm{h}$ & $\begin{array}{l}8 \mathrm{~km} / \mathrm{h} \text { and } \\
2.5 \% \text { gradient }\end{array}$ \\
\hline $\begin{array}{l}\text { SIR } \\
\text { Pre }\end{array}$ & $159(17)$ & $170(14)$ & $182(14)$ \\
$\quad$ Post & $160(13)$ & $168(16)$ & $177(13)$ \\
CCE & & & \\
$\quad$ Pre & $154(17)$ & $163(19)$ & $174(17)$ \\
$\quad$ Post & $156(16)$ & $166(18)$ & $171(18)$ \\
C Pre & $156(14)$ & $166(15)$ & $173(16)$ \\
$\quad$ Post & $154(11)$ & $160(14)$ & $168(17)$ \\
\hline
\end{tabular}

Values are mean (SD). SIR, sprint interval running; CCE, continuous cycle ergometry; C, control. No significant difference between groups $(p>0.05)$.

Table 4 Submaximal $\dot{V}_{E}$ (litres/min) at the first three stages of the treadmill test before (Pre) and after (Post) training.

\begin{tabular}{clll}
\hline & $7 \mathrm{~km} / \mathrm{h}$ & $8 \mathrm{~km} / \mathrm{h}$ & $\begin{array}{l}8 \mathrm{~km} / \mathrm{h} \text { and } \\
2.5 \% \text { gradient }\end{array}$ \\
\hline $\begin{array}{llll}\text { SIR } \\
\quad \text { Pre }\end{array}$ & $30.2(8.0)$ & $35.8(7.4)$ & $37.9(8.2)$ \\
$\quad$ Post & $31.3(7.6)$ & $33.8(9.6)$ & $37.4(9.3)$ \\
CCE & & & \\
$\quad$ Pre & $28.0(8.5)$ & $30.1(9.5)$ & $31.9(11.2)$ \\
$\quad$ Post & $27.9(6.1)$ & $29.7(9.7)$ & $32.8(8.2)$ \\
C Pre & $33.2(7.1)$ & $31.4(11.6)$ & $37.7(10.5)$ \\
$\quad$ Post & $30.4(6.4)$ & $33.9(9.0)$ & $35.8(9.0)$ \\
\hline
\end{tabular}

Values are mean (SD). SIR, sprint interval running; CCE, continuous cycle ergometry; C, control. No significant difference between groups $(p>0.05)$. 


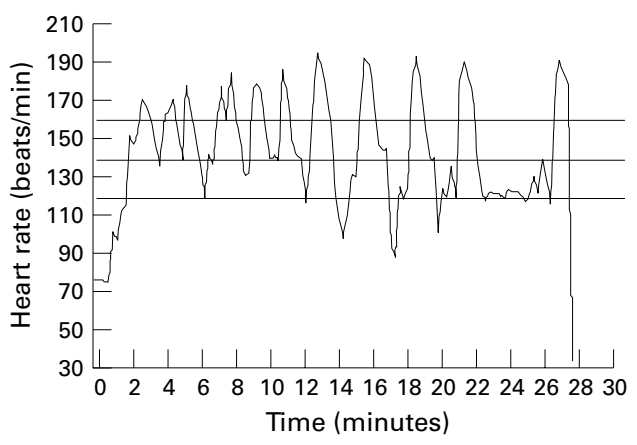

Figure 1 A typical heart rate during one of the sprint interval running training sessions.

the submaximal heart rate responses before and after training. No significant changes were found in the first three stages of the treadmill test $(p>0.05)$. Table 4 shows the submaximal $\dot{V} E$ scores before and after training. No significant changes were found in any group $(\mathrm{p}>0.05)$.

The distance run by the boys in the SIR group steadily increased as the training programme progressed. The mean total distance run for weeks 1 and 2 was 508 (32) m compared with 480 (30) $\mathrm{m}$ in weeks 3 and 4, 515 (33) $\mathrm{m}$ in weeks 5 and 6 , and $540(33 \mathrm{~m})$ in weeks 7 and 8 . The mean decrease from weeks 1 and 2 to 3 and 4 is a result of the inclement weather, which meant that three of the training sessions had to be performed in the school gymnasium; although the heart rate data and duration of the sprints were similar, the shortened running lanes dictated more turns and a slight loss of speed and therefore a reduced total distance run. The heart rate data were consistent throughout the SIR training period (mean 10 second sprints, 184 (8) beats/ min, mean 30 second sprints, 193 (9) beats/ $\mathrm{min}$ ) and were higher than the heart rate maintained by the CCE group. Figure 1 represents a typical example of one subject's heart rate data during an SIR training session.

\section{Discussion}

The key findings of this study are the non-significant effects of both a sprint interval running and a continuous cycle ergometer training programme on maximal and submaximal measures of aerobic performance.

The results of the study must be placed in the context of its strengths and limitations, as training studies with young children are notoriously difficult to perform and the existing literature is limited. Training programme durations are normally about eight weeks as in this study, although a longer programme may be more revealing. However, maintaining the interest of children in training programmes is difficult, and attrition rates as high as $63 \%$ have been reported. ${ }^{4}$ The attrition rate in the present study of $13 \%$ compares favourably with the literature and the attrition was due to factors external to the programme. Few studies have monitored objectively the intensity of their training programmes. In this study, exercise heart rates were rigorously monitored. Sample sizes tend to be small, and although the samples reported here are among the largest in the literature, given the small effect size the chance of type II errors must be acknowledged. Finally, very few studies define objectively the maturity status of their subjects. In this study all subjects were classified as Tanner stage one according to both genitalia and pubic hair development ${ }^{14}$ by an experienced nurse.

The peak $\dot{\mathrm{V}}_{2}$ values before training, which were not significantly different between the groups, compare favourably with those in the literature. ${ }^{515}$ Mean peak $\dot{\mathrm{V}}_{2}$ increases after the training period ranged from $2.6 \%$ in the control group, through $3.8 \%$ in the SIR group, to $7.2 \%$ in the CCE group, but no significant effects could be attributed to training. The highest percentage improvements, $25 \%$ and $22 \%$ in absolute and relative peak $\dot{\mathrm{VO}}_{2}$ respectively, were found in one subject in the CCE trained group; however, there were large individual variations in responses to training. These findings are in conflict with several other studies in which young boys were trained, ${ }^{17} 16$ and it is not readily apparent why no significant changes in peak $\mathrm{VO}_{2}$ were observed. However, the present results support the work of Gilliam and Freedson ${ }^{4}$ who were unable to elicit increases in the peak $\dot{\mathrm{V}}_{2}$ of 11 children 8 years of age who trained four times a week for 12 weeks, and they are in accord with the hypothesis of Gilliam and Freedson ${ }^{4}$ of a maturational factor playing a role in determining physiological change.

High levels of habitual physical activity have been proposed $^{17}$ as a reason for blunted training responses in young children. Previous work with boys from the same school as this study suggests that habitual physical activity is highly unlikely to have influenced peak $\dot{\mathrm{V}}_{2}{ }^{18}$ Data from British school aged children also confirm the lack of a significant relation between physical activity and both aerobic and anaerobic performance. ${ }^{19}$ This study showed that a five minute period of moderate activity (defined as heart rate $>139$ beats $/ \mathrm{min}$ ) was common in boys, but longer moderate to vigorous sustained periods of activity were seldom experienced. This observation was interpreted as reflecting the fact that few young children experience physical activity of the frequency, intensity, and duration likely to increase either anaerobic or aerobic performance. Falgairette et $a l^{20}$ also investigated the effects of physical activity on maximal oxygen uptake in active and non-active prepubertal boys. They concluded that active boys did not differ in aerobic performance when compared with non-active boys, despite a significant difference in physical activity.

It has been suggested ${ }^{10}$ that the relatively high initial aerobic fitness levels found in children may mitigate against training induced improvements. However, the initial peak $\dot{\mathrm{V}}_{2}$ of the present subjects was very similar to that of subjects in studies in which significant increases in peak $\dot{\mathrm{V}}_{2}$ were observed following training. ${ }^{17}{ }^{16}$ Some authors ${ }^{521}$ have suggested that exercise intensity appears to be the fundamental influence on the magnitude of training response. However, data are sparse and diffi- 
cult to interpret, as there is considerable variation in the intensity of training in many programmes and different protocols have been employed.

Savage et $a l^{1}$ scheduled training sessions three times a week for 11 weeks for the purpose of examining the effect of endurance training on serum lipids in prepubescent boys and adult men. Both boys and men were allocated to high intensity, low intensity, and control groups. The average heart rate of the boys during the sessions was $67.7 \%$ of maximum heart rate for the low intensity group (equivalent to $40 \% \dot{\mathrm{V}}_{2} \mathrm{MAX}$ ) for the high intensity group. Significant increases in peak $\dot{\mathrm{V}}_{2}(4.7 \%$ for boys) were limited to the high intensity training groups but did not differ between men and boys. Lussier and Buskirk ${ }^{7}$ implemented a four times a week, 45 minutes per session, 12 week training programme with a group of 26 children. During the training, the experimental group $(n=12)$ maintained their heart rate at "about $92 \%$ of heart rate maximum" ( $p$ 737) during each session. This target heart rate was in the range $75-85 \% \dot{V}_{\mathrm{O}_{2}} \mathrm{MAX}$. After the 12 week programme $\dot{\mathrm{V}}_{2} \mathrm{MAX}$ relative to body mass had significantly increased (average $6.8 \%$ ) in the trained group but not in the control group. Weltman et $a l^{2}$ implemented a 14 week, four times a week resistance training programme and showed an increase in mass related peak $\dot{\mathrm{V}}_{2}$ of $13.7 \%$ for the experimental group of prepubertal boys $(n=16)$.

The optimal intensity, frequency, and duration of exercise for peak $\dot{\mathrm{V}}_{2}$ improvements is unknown but the training programme of the CCE group was in accord with current recommendations. ${ }^{22}$ The 10 second and 30 second work intervals of the SIR group were chosen to mirror the intermittent nature of children's physical activity patterns ${ }^{13}$, as well as to contrast with a conventional aerobic training programme. ${ }^{5}$ Hence the short work intervals enabled the SIR group to maintain a high intensity of effort. In addition, the short high intensive sprint followed by a passive recovery simulated conditions that replicate many daily habitual physical activities - for example, playing football or chase in the school playground. The training programme was similar to that used by Grodjinovsky et al who used three sprints of $40 \mathrm{~m}$ followed by three sprints of 150 $\mathrm{m}$ and increased the number of runs every two weeks. On the basis of the recorded heart rate data and distance run, we are confident that near maximum effort was achieved during the SIR programme. Despite the high training intensity, no significant improvement in peak $\dot{\mathrm{V}} \mathrm{O}_{2}$ was observed with the SIR group. Based on the heart rate data used to monitor the SIR group and subjective observation of intensity by the researchers, it is difficult to envisage the children working more intensely.

Longitudinal studies in which the effects of training on aerobic performance were observed have produced conflicting results. Kobayashi et $a l^{23}$ and Mirwald et $a l^{24}$ both suggest that maximal aerobic power cannot be increased with training during the prepubertal years. The most recently published longitudinal investiga- tion, the training of young athletes (TOYA) study, found training to increase the aerobic power of prepubertal children above the normal increase attributable to age, physical growth, and maturation. ${ }^{25}$ The TOYA study investigated training effects on a total of 453 athletes from four sports, tennis, swimming, soccer, and gymnastics. When age, stature, and body mass were controlled, $\dot{\mathrm{V}}_{2} \mathrm{MAX}$ significantly increased with pubertal status. Girls also showed a similar trend, but the significant increases in $\dot{\mathrm{V}}_{2}$ shown by the boys towards the end of puberty were not replicated by the girls. The authors suggested that, in the more aerobic sports such as swimming, the aerobic training effect was most noticeable. It was also reported that, in the four sports studied, the type of sports training influenced the development of aerobic power. However, the TOYA study was an observational study and therefore was not able to manipulate the training programmes of the different sports.

Despite the general trend of lower submaximal heart rate data after training in this study, the large group variation in heart rate data may have masked any individual training adaptations. Decreases in submaximal heart rate with training have been found in other studies. ${ }^{26}{ }^{27} \mathrm{In}$ the study by Lussier and Buskirk, ${ }^{7}$ submaximal indices of heart rate were determined at 53\%, $68 \%$, and $80 \%$ of $\dot{\mathrm{V}}_{2} \mathrm{MAX}$. Significant differences following training were found between the exercise and control group for heart rate at $40 \%$ and $80 \% \dot{\mathrm{V}} \mathrm{O}_{2} \mathrm{MAX}$. However, these results should be tempered with the fact that there were no apparent changes in the exercise group for submaximal cardiac output or stroke volume. Moreover, most other studies have shown few if any submaximal changes with training. ${ }^{5}$ Mayers and Gutin ${ }^{28}$ attribute this to the nature of short term training versus training that has continued for several years. These authors suggest that adaptive changes after 10 weeks of training will occur both at the local and systemic level, but the relative changes will be small in comparison with training programmes of six months or more.

Given the lack of a change at maximal exercise intensities, it has been suggested that submaximal changes may be more likely to occur than maximal changes, although these may be related to changes in running economy. Indeed, Bar- $\mathrm{Or}^{29}$ has speculated that a programme based on exclusively improving "running style might in itself improve the running performance of children" ( $p$ 76). It is possible that, had the CCE training group trained by running, submaximal changes may have been more evident. However, an important feature of any training programme is how precisely the intensity is determined. The use of cycle ergometry for the continuous exercise group enabled us accurately to monitor and record the intensity during each session.

In conclusion, neither submaximal nor maximal aerobic variables were improved by an eight week training programme. The results of this study support the view that prepubertal boys have a blunted response to eight weeks training. ${ }^{30}$ Much more research with well 
defined samples and controlled training programmes of longer duration intensity is necessary to clarify the effects of exercise training on prepubertal boys.

1 Savage MP, Petratis MM, Thomson WH, et al. Exercise training effects on serum lipids of prepubescent boys and training effects on serum lipids of prepubescent boy

2 Weltman A, Janney J, Rians CB, et al. The effects of hydraulic-resistance strength training on serum lipid levels hydraulic-resistance strength training on serum lipid le

3 Becker DM, Vaccaro P. Anaerobic threshold alterations caused by endurance training in young children. $\mathcal{F}$ Sports Med 1983;23:445-9.

4 Gilliam TB, Freedson PS. Effects of a 12 week school physical fitness program on peak $\mathrm{VO}_{2}$, body composition and blood lipids in 7 to 9 year old children. 7 Sports Med 1980 $1: 73-8$

5 Armstrong N, Welsman J. Young people and physical activity. Oxford: University Press, 1990:122-36.

6 Pate RR, Ward DS. Endurance exercise trainability in children and youth. In: Grana WA, Lombardo JA, Sharkey $\mathrm{BJ}$, et al, eds. Advances in sports medicine and fitness. Chicago: Year Book Publishers, 1990;3:37-55.

7 Lussier L, Buskirk ER. Effects of an endurance training regimen on assessment of work capacity in prepubertal regimen on assessment of work capacity in p
children. Ann N Y Acad Sci 1990;30:734-47.

8 Grodjinovsky A, Inbar O, Dotan R, et al. Training effect on the anaerobic performance of children as measured by the the anaerobic performance of children as measured by the Wingate anaerobic test. In: Berg K, Eriksson B, eds. Children and exercise IX. Baltimore: University Park Press, 1980:139-45.

9 Katch VL. Physical conditioning of children. If Adolesc Health Care 1983;3:241-6.

10 Shephard RJ. Effectiveness of training programmes for prepubescent children. Sports Med 1992;13:194-213.

1 Armstrong N, Balding J, Gentle P, et al. Patterns of physical activity among 11 to 16 year old British children. $B M \mathcal{F}$ 1990;301:203-5.

12 Bailey RC, Olson J, Pepper SL, et al. The level and tempo of children's physical activities: an observational study. Med Sci Sports Exerc 1995;27:1033-41.

13 Armstrong N, Kirby B, McManus A, et al. Aerobic fitness of prepubescent children. Ann Hum Biol 1995;22:435-76.

14 Trepubescent children. Ann Hum Browth at adolescence. 2nd ed. Oxford: Blackwell Scientific Publications, 1962.
15 Armstrong N, Welsman J. Assessment and interpretation of aerobic fitness in children and adolescents. Exerc Sport Sci Rev 1994;22:435-76.

16 Rotstein A, Dotan R, Bar-Or O, et al. Effect of training on anaerobic threshold, maximal aerobic power and anaerobic performance of preadolescent boys. Int $\mathcal{F}$ Sports Med 1986; 7:281-6

17 Krahenbuhl GS, Skinner JS, Kohrt WM. Developmental aspects of maximal aerobic power in children. Exerc Sport Sci Rev 1985;13:503-38.

18 Armstrong N, McManus A, Welsman J, et al. Physical activity patterns and aerobic fitness among pre-pubescents. European Physical Education Review 1996;2:19-29.

19 Armstrong N, Welsman J, Kirby B. Physical activity, peak oxygen uptake and performance on the Wingate anaerobic
test in 12-year-olds. Acta Kinesiologica 1998;3:7-21.

20 Falgairette G, Duche P, Bedu M, et al. Bioenergetic characteristics in prepubertal swimmers. Comparison with active

21 Washington RL. Anaerobic threshold in children. Pediatric Exercise Science 1989;1:244-56.

22 American College of Sports Medicine. Opinion statement on physical fitness in children and youth. Med Sci Sports Exerc 1988;20:422-3.

23 Kobayashi K, Kitamura K, Miura M, et al. Aerobic power as related to body growth and training in Japanese boys: a longitudinal study. F Appl Physiol 1978;44:666-72.

24 Mirwald RL, Bailey DA, Cameron N, et al. Longitudinal comparison of aerobic power on active and inactive boys aged 7 to 17 years. Ann Hum Biol 1981:8:405-14.

25 Baxter-Jones A, Goldstein H, Helms P. The development of aerobic power in young athletes. F Appl Physiol 1993;75: 1160-7.

26 Bar-Or O, Zwiren LD. Physiological effects of increased frequency of physical education classes and of endurance conditioning on 4- to 10-year-old girls and boys. In: Bar-Or O, ed. Pediatric work physiology. Natanya: Wingate Institute, 1973:183-9.

27 Stewart KJ, Gutin B. Effects of physical training on cardiorespiratory fitness in children. Res $Q 1976 ; 47: 110$ 20.

28 Mayers N, Gutin B. Physiological characteristics of elite prepubertal cross-country runners. Med Sci Sports Exerc 1979;11:172-6.

29 Bar-Or O. Trainability of the prepubescent child. Physician and Sportsmedicine 1989;17:228-36.

30 Rowland T. The aerobic trainability of athletic and non-athletic children. In: Froberg $\mathrm{K}$, Lammert $\mathrm{O}, \mathrm{St}$ Hansen $\mathrm{H}$, et al, eds. Exercise and fitness: benefits and risks. Odense: University Press, 1997:183-92.

Applications are invited for the post of:

\section{EDITOR}

\section{British Journal of Sports Medicine}

Journal of the British Association of Sport and Exercise Medicine

Specialists in any branch of sports and exercise medicine are invited to apply for the post of Editor. Please send a letter of application, curriculum vitae, a short statement about the strengths and weaknesses of British fournal of Sports Medicine, and your proposed editorial policy.

Full editorial support will be provided and it is envisaged that the editor will need to devote about $1 / 2$ day per week to the journal.

British fournal of Sports Medicine is international and we seek applicants world wide. Joint applications from two or more candidates wishing to act as co-Editors will be considered.

Closing date is 30 June 2000. Interviews will be held in July 2000 to enable the successful candidate(s) to take up the post in the fourth quarter of 2000.

Details of the post can be discussed with the current editor (Professor Domhnall MacAuley, email: DC.MacAuley@ulst.ac.uk) or Mrs Alex Williamson, to whom applications should be sent. A job description is available on request.

Mrs Alex Williamson, BMJ Publishing Group, BMA House, Tavistock Square, London WC1H 9JR, UK. Telephone +44 (0)20 7383 6169; Fax: +44 (0)20 7383 6668; email: awilliamson@bmjgroup.com. 